\title{
Effects of dilated cardiomyopathy on the diaphragm in the Syrian hamster
}

\author{
G. Stassijns***, G. Gayan-Ramirez*, P. De Leyn+, V. de Bock*, \\ R. Dom ${ }^{\S}, \mathrm{R}$. Lysens**, M. Decramer*
}

Effects of dilated cardiomyopathy on the diaphragm in the Syrian hamster. G. Stassijns, G. Gayan-Ramirez, P. De Leyn, V. de Bock, R. Dom, R. Lysens, M. Decramer. (C)ERS Journals Ltd 1999.

ABSTRACT: This study aimed to elucidate changes in respiratory muscles and their mechanism in cardiomyopathy. The contractile properties and histology of the diaphragm, as well as serum levels of insulin-like growth factor (IGF)-1, were examined in 10 hamsters with idiopathic dilated cardiomyopathy (CM) and 10 controls.

At 28 weeks, body weight in $C M$ was reduced compared with controls $(114 \pm 10$ versus $144 \pm 14 \mathrm{~g}, \mathrm{p}<0.0001)$. The ratio of diaphragm to body weight was significantly higher in $C M$ than in controls $(0.228 \pm 0.015$ versus $0.182 \pm 0.017, p<0.0001)$. In vitro, maximal diaphragmatic twitch $\left(303 \pm 63\right.$ versus $\left.455 \pm 119 \mathrm{~g} \cdot \mathrm{cm}^{-2}\right)$ and tetanic tensions $\left(1,555 \pm 369\right.$ versus $\left.2,204 \pm 506 \mathrm{~g} \cdot \mathrm{cm}^{-2}\right)$ were significantly lower in CM than in controls $(\mathrm{p}<0.005)$. The half-relaxation time was significantly shorter in $\mathrm{CM}(19 \pm 1 \mathrm{~ms})$ than in controls $(24 \pm 3 \mathrm{~ms}, \mathrm{p}<0.0005)$. Fatiguability at $25 \mathrm{~Hz}$ was significantly less in $\mathrm{CM}$ $(28 \%)$ than in controls $(42 \%, p<0.0001)$. Diaphragm and gastrocnemius adenosine triphosphatase staining showed type I fibre atrophy in CM, associated with an increase in the number of type $I$ fibres in the diaphragm. Histological examination of both muscles revealed an abnormal muscular pattern. Finally, serum levels of IGF-1 were $47 \%$ lower in the $C M$ group than in controls $(p<0.0001)$ and were clearly related to the changes in the contractile properties and histology of the diaphragm.

In conclusion, cardiomyopathy in hamsters: 1) depressed the force-generating capacity and shortened the relaxation of the hamster diaphragm; 2) induced type I fibre atrophy in combination with a myopathic pattern; and 3) was associated with a significant reduction in serum levels of insulin-like growth factor-1, related to the diaphragmatic changes. Whether these changes are primary myopathic or secondary to heart failure remains to be elucidated.

Eur Respir J 1999; 13: 391-397.
*Respiratory Muscle Research Unit, Laboratory for Pneumology and Respiratory Division, **Dept of Physical Medicine and Rehabilitation, ${ }^{+}$Division of Thoracic Surgery, and ${ }^{\S}$ Dept of Neuropathology, University Hospitals Katholieke Universiteit Leuven, Leuven, Belgium.

Correspondence: M. Decramer

Respiratory Division, University Hospital Herestraat 49

3000 Leuven

Belgium

Fax: 3216347126

Keywords: Cardiac failure cardiomyopathy

contractile properties diaphragm

histology

Received: November 201997 Accepted after revision October 11998

Supported by Fonds voor Wetenschappelijk Onderzoek-Vlaanderen grant No. G0189. 97 and Astra Pharmaceuticals.
Muscle fatigue and dyspnoea are the major symptoms reported by patients with congestive heart failure (CHF) [1]. Alterations in the contractile properties, histology and biochemistry of both peripheral and respiratory muscles [1-3] have been proposed as significant contributors to these symptoms. The former include: reduced endurance, variable fibre atrophy, increased percentage of glycolytic type IIb fibres and reduced oxidative enzymatic activity. Different forms of heart failure appear to affect the skeletal muscles in different ways [4]. Therefore, a model of cardiomyopathy $(\mathrm{CM})$ was studied to determine how this type of heart failure affects the diaphragm and peripheral muscles. The model of dilated CM in the Syrian hamster was used since this has been suggested to be more closely related pathophysiologically to human CM than the hypertrophic model [5]. In the dilated model, only data on diaphragm force are available, whereas skeletal muscle histology has only been documented in a model of hypertrophic CM [6]. Diaphragm fatigue has not been described in cardiomyopathic hamsters.

Serum levels of insulin-like growth factor (IGF)-1 were also determined since they have been found to be signi- ficantly reduced in patients with $\mathrm{CHF}$ [7]. In addition, IGF1 plays an important role in skeletal muscle protein anabolism and growth [8] and could, therefore, be involved in the skeletal muscle alterations seen in $\mathrm{CM}$ and CHF.

The purpose of the present study was to examine the effects of CM on force generation and fatiguability as well as on the histopathology of the diaphragm. A further aim was to evaluate whether the finding of reduced serum levels of IGF-1 in patients [7] was reproducible in animals with $\mathrm{CM}$ and whether reduced IGF-1 levels were related to the diaphragmatic abnormalities.

\section{Materials and methods}

\section{Animals and study design}

All experiments were conducted with 28 -week-old male Syrian hamsters, with a body weight of $144 \pm 14 \mathrm{~g}$ (controls) and $114 \pm 10 \mathrm{~g}(\mathrm{CM})$. The animals were obtained from Bantin and Kingman breeders (Fitchburg, MA, USA). Ten 
CM (Bio TO2 strain) and 10 healthy age-matched control golden Syrian hamsters (F1B) were used for the study.

\section{In vitro study}

The Syrian hamsters were anaesthetized with sodium pentobarbital (Nembutal, $60 \mathrm{mg} \cdot \mathrm{kg}^{-1}$ i.p.) and then tracheostomized to insert a tracheal cannula (polyethylene tubing, PE-200). They were mechanically ventilated during the time of dissection (Harvard pump respirator, South Natick, MA, USA), with an $\mathrm{O}_{2}$-enriched gas mixture, because the laparotomy, performed to remove the diaphragm, made spontaneous breathing impossible. Following this procedure optimal oxygenation and viability of the diaphragm were obtained.

In vitro contractile properties. After laparotomy, the removed diaphragm was placed in an aerated and cooled Krebs solution containing (in mmol. $\mathrm{L}^{-1}$ ): $\mathrm{NaCl} 137, \mathrm{KCl}$ 4, $\mathrm{CaCl}_{2} 2, \mathrm{MgCl}_{2} 1, \mathrm{KH}_{2} \mathrm{PO}_{4} 1, \mathrm{NaHCO}_{3} 12$ and glucose 6.5. Two small strips of the diaphragm were then dissected as described in detail elsewhere [9]. Bundles, studied in pairs, were mounted vertically in a tissue bath containing Krebs solution aerated with $95 \% \mathrm{O}_{2} / 5 \% \mathrm{CO}_{2}$ at a temperature of $37^{\circ} \mathrm{C}$ and stimulated as described elsewhere [9]. Stimulations were performed with 0.2-ms supramaximal square-wave pulses and a train duration of $250 \mathrm{~ms}$. The muscles were first placed at the length at which maximal isometric twitch tension was obtained. This was called the optimal length $\left(L_{0}\right)$. The signals were amplified and recorded on computer via analogueto-digital conversion (DT 2801-A) using Labdat software (Labdat/Anadat, RHT-Infodat, Montreal, Canada). Measurements were made with Anadat.

After a 15 -min thermoequilibration period, the following measurements were successively performed at $L_{0} .1$ ) Twitch characteristics were determined from two successive measurements: the highest value of peak twitch force $(P \mathrm{t})$ and its corresponding time to peak tension (TPT) and half-relaxation time $(1 / 2 \mathrm{RT})$ were used for further analysis. 2) Fused tetanic force $\left(P_{\mathrm{o}}\right)$ was recorded using 160 $\mathrm{Hz}$ stimulation. Two successive stimulations were applied at 2-min intervals. The highest value was taken for further analysis. 3) The force-frequency relationship was measured with 2-min intervals between the stimulations, using the following order of frequencies: $25,160,50,160,80$, 160,120 and $160 \mathrm{~Hz}$ (modified from [10]). 4) Fatiguability of the bundles was assessed by using a fatigue run consisting of repeated $25-\mathrm{Hz}$ stimulations in trains of 330ms duration with one each $3 \mathrm{~s}$ during 5 min. 5) Deterioration of the preparation due to the duration of the protocol was evaluated by measuring the tension developed at $160 \mathrm{~Hz}$ after each stimulus during the forcefrequency protocol.

After completion of the experiment the bundle length, thickness and width were measured at $L$ o using fine calipers. The bundle was dried and fresh muscle weight was determined. Cross-sectional area (CSA) was obtained by dividing weight by specific density (1.056) and muscle length obtained at $L$ o. All forces were expressed per unit CSA [10]. The twitch-to-tetanus ratio $\left(P_{\mathrm{t}} / P_{\mathrm{o}}\right)$ was also calculated for each muscle bundle.

Heart, liver, diaphragm and gastrocnemius were weighed after they had been trimmed and dried.

\section{Histological and histochemical analysis}

The gastrocnemius and diaphragm muscles were folded, cut transversely and placed in tissue glue (Tissue-Tek, Elkard, IN, USA) on a cork, such that the longitudinal axis of fibres was perpendicular to the cork. Each muscle specimen was immediately immersed in isopentane cooled with liquid $\mathrm{N}_{2}$. Subsequently, all muscle biopsies were cut with a cryostat kept at $-20^{\circ} \mathrm{C}$ to obtain cross-sections of 10 $\mu \mathrm{m}$. Two sections of each muscle were stained using haematoxylin and eosin (H\&E). The other serial sections were stained for myofibrillar adenosine triphosphatase (ATPase) after acid preincubation at $\mathrm{pH} 4.5$ and 4.3. For all muscles studied, slides at $\mathrm{pH} 4.5$ offered the better separation of the two fibre types and were, therefore, used for morphometric analysis. Because of the uniform histochemical staining profile of type II fibres in CM no attempt was made to subclassify type II fibres (as IIa, IIb, IIc, or IIx), especially as such classification in hamsters is controversial. According to LeWIS et al. [11] there is no objective basis (i.e. on the basis of densitometric analysis) for such classification in the hamster diaphragm, although one study, using dystrophic hamsters, subclassified the type II fibre types [6]. Varied stain intensity is, however, mentioned in the diaphragm sections of dystrophic hamsters, making interpretation less reliable. Quantitative measurements of myopathic features were made after H\&E staining of the diaphragm, using a microscope (Leitz Laborhama S., Wetzlar, Germany) at $20 \times$ magnification (objective), connected to a computerized image analysis system (Quantimet 500; Leica, Cambridge, UK). The following variables were determined in both CM and control animals: number of necrotic cells, number of cells with $>5$ nuclei and number of cells with central nuclei. Each was expressed as a percentage of the total number of cells.

\section{Fibre size and proportion}

Morphometric examination was performed by microscopy at $20 \times$ magnification as described above. A minimum of 150 fibres of each muscle was used to calculate the proportion and CSA of all fibre types. Boundaries of individual muscle fibres were delineated and fibre CSA were determined from the number of pixels within the outlined fibre. At the same time, fibre diameters, defined as orthoferret (longest length perpendicular to fibre long axis), were automatically determined. In addition, for the diaphragm the CSA were corrected for the shortening occurring from $L \mathrm{o}$, the mean correction factor being similar in control and CM animals (pooled value for the diaphragm $=1.77 \pm 0.35$ ). For the gastrocnemius such correction was not performed since the $L$ o of peripheral muscles has generally been found to be between 100 and $120 \%$ of their resting length, with the resting length corresponding to the excised length [12].

Measurement of serum levels of insulin-like growth factor-1

Blood was sampled immediately after dissection of the heart from the thorax. Serum was obtained by centrifugation at $1,600 \times g$ for $10 \mathrm{~min}$ at $4{ }^{\circ} \mathrm{C}$ and stored at $-20^{\circ} \mathrm{C}$. 
IGF-1 was measured in acid-ethanol-extracted sera by radioimmunoassay as described previously [13], with the use of a guinea-pig polyclonal antiserum (Ciba-Geigy, Basel, Switzerland).

\section{Data analysis}

The statistical analysis was performed using the SAS statistical package (SAS Institute, Cary, NC, USA). Data from the two diaphragm strips obtained from each hamster were averaged for further statistical analysis. Differences between means of controls and CM hamsters were assessed using the Student's t-test. The Student's t-test was also used to evaluate histochemical findings. Correlations were determined using Spearman's rank product correlation.

Statistical significance was defined as a two-tailed pvalue $<0.05$. Data are expressed as means \pm SD.

\section{Results}

\section{Body, muscle and organ weight}

At 28 weeks, body weight in the CM hamsters was significantly reduced in comparison with the controls: CM $114 \pm 10$ versus controls $144 \pm 14 \mathrm{~g}(\mathrm{p}<0.0001)$.

A significantly lower heart (16\%) and liver weight (24\%) was seen in the CM hamsters than in the control hamsters $(p<0.0001$ and $p<0.005$, respectively). When expressed as a percentage of the body weight, however, there were no significant differences between the groups.

The absolute weight of the diaphragm was similar in both groups (pooled value: $260 \pm 31 \mathrm{mg}$ ), while after normalization for body weight, the diaphragm to body weight ratio was significantly higher in the CM group $(0.228 \pm$ $0.015)$ than in the controls $(0.182 \pm 0.017, \mathrm{p}<0.0001)$. Gastrocnemius weight was significantly lower in the CM group ( $225 \pm 23$ versus $263 \pm 17 \mathrm{mg}, \mathrm{p}<0.005)$. However, after normalization for body weight gastrocnemius weight was increased (0.196 in CM versus 0.184 in controls, $\mathrm{p}<$ $0.05)$.

The thickness and width of the dissected diaphragm bundles were comparable in the two groups. The length of the diaphragm bundles, however, was slightly but significantly smaller in the CM group $(13.5 \pm 1.2$ versus $14.8 \pm$ $1.1 \mathrm{~mm}, \mathrm{p}<0.05)$.

\section{Contractile properties of the diaphragm}

$P \mathrm{t}$ and $P_{\mathrm{o}}$ were both comparably reduced in the CM group (by $34 \%$ and $29 \%$, respectively, $\mathrm{p}<0.005$ ), so that no significant changes were seen in the twitch to tetanus ratio, $P \mathrm{t} / P_{\mathrm{o}}$ (table 1$)$. No statistically significant difference in TPT was seen between the groups. Conversely, the 1/2 RT was significantly reduced by $19 \%$ in the CM group $(\mathrm{p}<0.0005)($ table 1$)$.

The force-frequency curve showed that the $\mathrm{CM}$ diaphragm bundles generated a significantly lower force
Table 1. - Diaphragm contractile properties in control and cardiomyopathic hamsters

\begin{tabular}{lcc}
\hline & $\begin{array}{c}\text { Control } \\
\text { Bio F1B }(\mathrm{n}=10)\end{array}$ & $\begin{array}{c}\text { Cardiomyopathy } \\
\text { Bio TO2 }(\mathrm{n}=10)\end{array}$ \\
\hline$P_{\mathrm{t} \mathrm{g} \cdot \mathrm{cm}^{-2}}$ & $455 \pm 119$ & $303 \pm 63^{+}$ \\
$P_{\mathrm{o} \mathrm{kg}} \cdot \mathrm{cm}^{-2}$ & $2.204 \pm 506$ & $1.555 \pm 369^{+}$ \\
$P_{\mathrm{t}} / \mathrm{O}_{\mathrm{o}}$ & $0.205 \pm 0.021$ & $0.199 \pm 0.025$ \\
$\mathrm{TPT} \mathrm{ms}$ & $22.6 \pm 1.2$ & $21.9 \pm 1.9$ \\
$1 / 2 \mathrm{RT} \mathrm{ms}$ & $24.0 \pm 2.8$ & $19.5 \pm 1.4^{\#}$ \\
\hline
\end{tabular}

$P_{\mathrm{t}}$ : twitch tension; $P_{\mathrm{o}}$ : tetanic tension; $P_{\mathrm{t}} / P_{\mathrm{o}}$ : twitch to tetanus ratio; TPT: time to peak tension; $1 / 2 \mathrm{RT}$ : half-relaxation time. ${ }^{+}$: $\mathrm{p}<0.005 ; \stackrel{\#}{ }: \mathrm{p}<0.0005$.

at all frequencies $(\mathrm{p}<0.0005)$ (fig. 1a). When expressed as a percentage of the interposed $160 \mathrm{~Hz}$ stimulations, the force response was still shifted to the right, reaching statistical significance at 25 and $50 \mathrm{~Hz}$ (fig. 1b).

The absolute values of decline in tension $(160 \mathrm{~Hz})$ during the force-frequency protocol were significantly different $\left(750 \pm 205\right.$ versus $500 \pm 225 \mathrm{~g} \cdot \mathrm{cm}^{-2}$, in the $\mathrm{CM}$ and control group respectively, $\mathrm{p}<0.05)$. When these values were expressed as percentage of $P_{\mathrm{o}}$, the decline in tension in the CM group was double that in the control group (48 \pm 11 versus $24 \pm 10 \%, \mathrm{p}<0.001$ ).

During the fatigue run, the $\mathrm{CM}$ diaphragm strips generated significantly lower force than did the control diaphragm, when force was expressed per unit CSA (fig. 2). Such an effect was already present at the beginning of the fatigue run and persisted during the whole protocol.
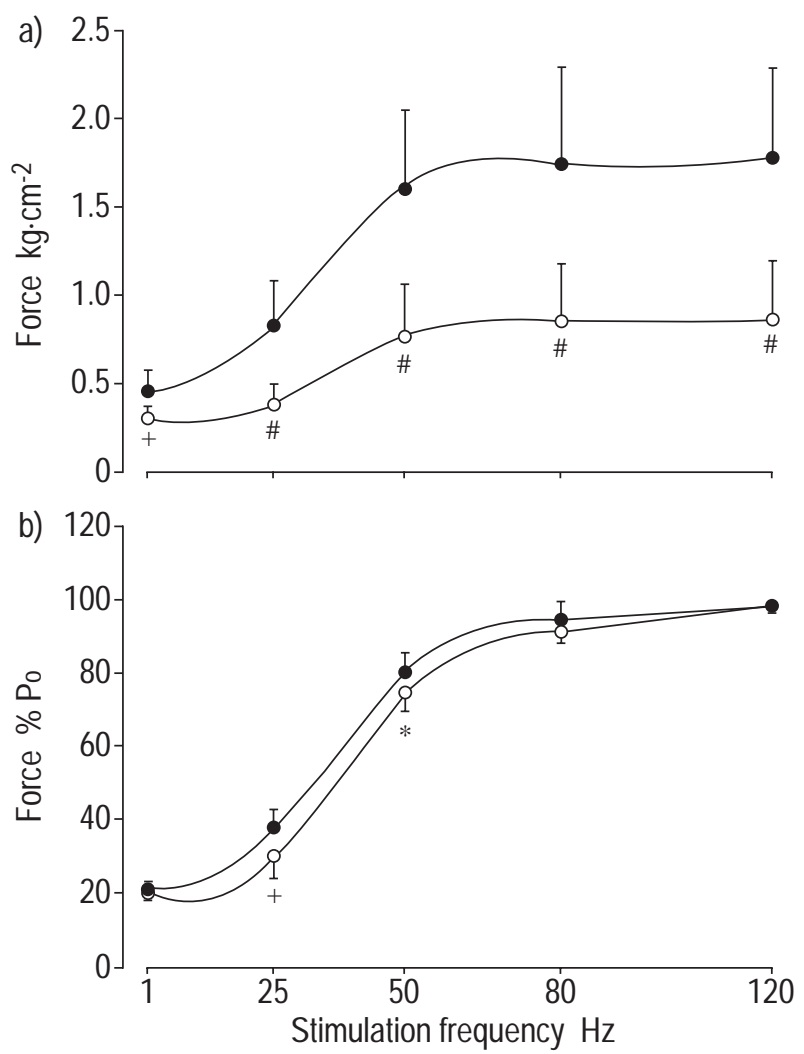

Fig. 1. - Force-frequency relationship expressed in a) absolute values and $b$ ) as a percentage of interposed $160 \mathrm{~Hz}$ stimulations in control ( and cardiomyopathic $(\bigcirc)$ hamsters. Po: tetanic tension. ${ }^{*}: \mathrm{p}<0.05 ;{ }^{+}: \mathrm{p}<$ $0.005 ;{ }^{\#}: \mathrm{p}<0.0005$. 

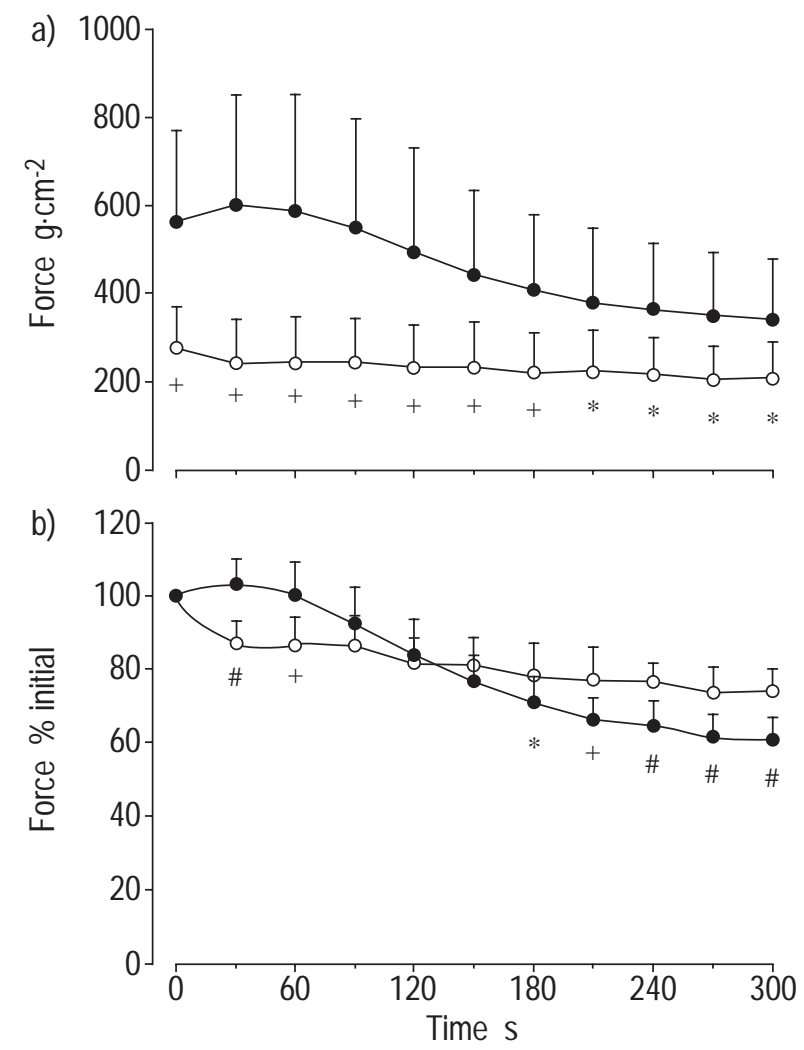

Fig. 2. - Fatigue curve expressed in a) absolute values and b) as a percentage of initial force in control $(0)$ and cardiomyopathic $(\bigcirc)$ hamsters. *: $\mathrm{p}<0.05{ }^{+}: \mathrm{p}<0.005 ;{ }^{\#}: \mathrm{p}<0.0005$.

The decline in force at the end of the fatigue run (fatiguability) was significantly less in the CM $(28 \%)$ than in the control group $(42 \%, \mathrm{p}<0.0001)$.

\section{Histology and histochemistry}

Typical histological features of H\&E-stained diaphragm slides of control and CM hamsters are shown in figure 3a and $b$, respectively. Quantitative analysis of the myopathic features in the diaphragm showed greater proportions of necrotic cells ( $12 \pm 3$ versus $2 \pm 1 \%$ ), of cells with $>5$ nuclei $(24 \pm 5$ versus $6 \pm 3 \%)$ and of cells with central nuclei (54 \pm 6 versus $10 \pm 3 \%$ ) in the CM hamsters than in controls $(p<0.0001$ for the three variables). H\&E staining of the gastrocnemius showed a comparable myopathic pattern. Histochemical analysis of the diaphragm after ATPase staining showed a significantly greater proportion of type I fibres (29 versus $38 \%, \mathrm{p}<0.005)$ in the CM group, with a moderate reduction in the proportion of type II cells ( 71 versus $62 \%, \mathrm{p}<0.005)$. ATPase stainings of the diaphragm are shown in representative control (fig. 4a) and CM (fig. 4b) hamsters. Conversely, in the CM group selective type I fibre atrophy was seen, as was evident from a significant reduction in CSA $(-29 \%, \mathrm{p}<0.005)$ (fig. $5)$ and in diameter $(-17 \%, p<0.0001)$, while type II fibre CSA and diameter remained unchanged. The total surface area taken up by type I fibres (defined as the mean surface area of type I fibres $\times$ mean proportion of type I fibres) was comparable in the two groups.
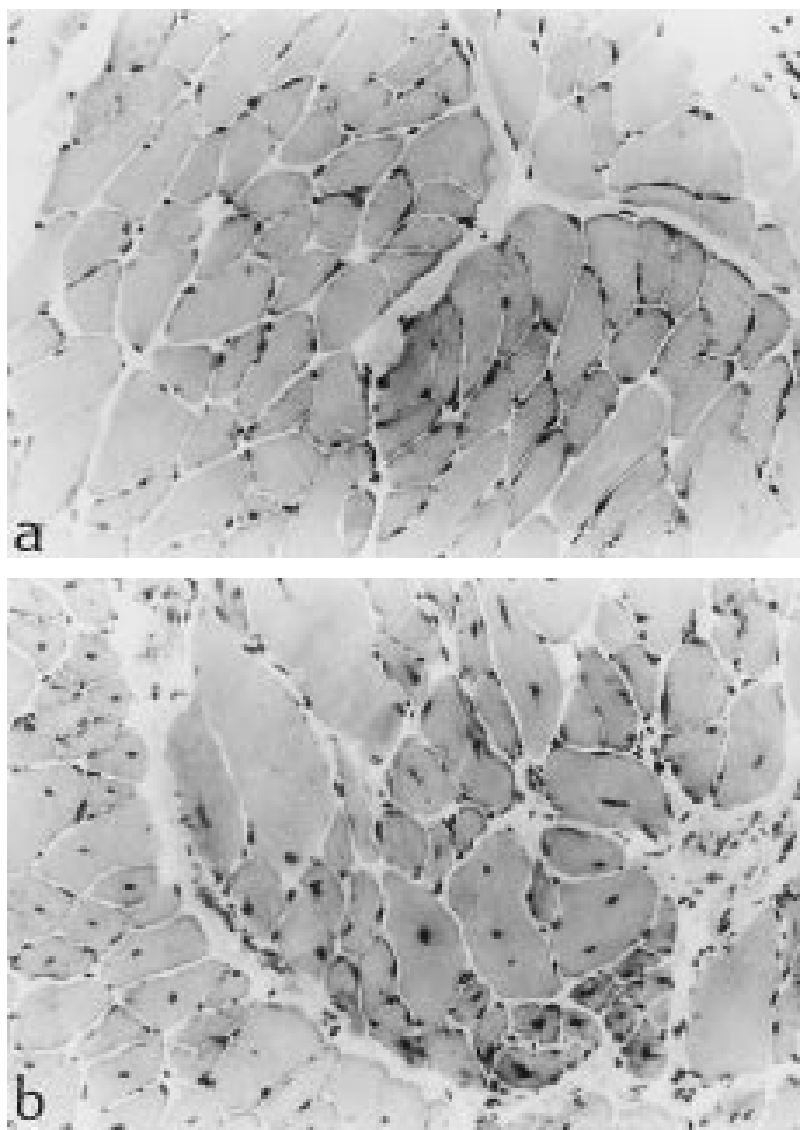

Fig. 3. - Haematoxylin and eosin (H\&E) staining of a) control and b) cardiomyopathic diaphragms from representative animals. Note the increase in the number of cells with central nuclei, the number of cells with more than five nuclei and the number of necrotic cells in (b). (Magnification $20 \times$ (objective).)

Histochemical analysis of the gastrocnemius also showed type I atrophy. CSA and diameter were significantly reduced in CM animals $(-24 \%$ and $-16 \%, \mathrm{p}<0.01$ and $\mathrm{p}<$ 0.05 , respectively). No fibre shift was seen. The dimensions of the type II fibres were unaltered.

\section{Serum levels of insulin-like growth factor-1}

A significant decrease of $47 \%$ in IGF-1 serum levels was apparent in the CM group $\left(204 \pm 38 \mathrm{ng} \cdot \mathrm{mL}^{-1}\right) \mathrm{com}$ pared with the controls $\left(383 \pm 43 \mathrm{ng} \cdot \mathrm{mL}^{-1}, \mathrm{p}<0.0001\right)$ (fig. $6)$. There were significant correlations for all animals (both groups taken together) between serum IGF-1 levels on the one hand and 1/2 RT and force during the forcefrequency curve of the diaphragm, on the other hand. The correlation coefficients were $r=0.70$ and $p<0.001$ for $1 / 2$ RT and $r$ ranging $0.57-0.62$ for forces during the forcefrequency curve, with $\mathrm{p}$ ranging from $<0.005$ at stimulation frequencies of 25,50 and $120 \mathrm{~Hz}$ to $<0.01$ at 80 $\mathrm{Hz}$. In addition, serum IGF-1 levels were inversely related to fatigue during the fatigue run $(r=-0.67, p=0.001$ at the end of the fatigue run). The type I fibre surface area of the diaphragm was significantly related to the IGF-1 serum levels $(\mathrm{r}=0.51, \mathrm{p}<0.05)$. 


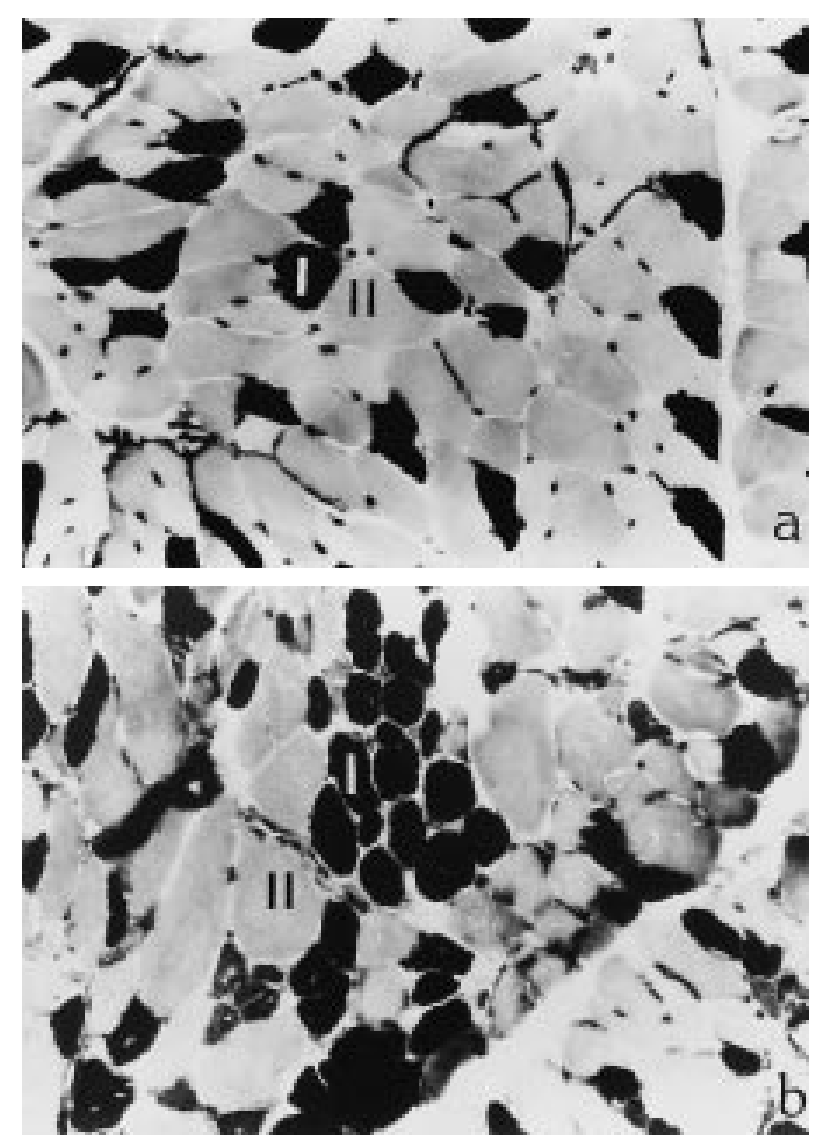

Fig. 4. - Adenosine triphosphatase staining of the diaphragm at $\mathrm{pH} 4.5$ in a) representative control and b) cardiomyopathic hamsters. Note the increase in the number of type I fibres and the atrophy of the type I fibres in (b). (Magnification $20 \times$ (objective).)

\section{Discussion}

In the present study, the force-generating capacity of the diaphragm was depressed in CM animals compared with controls, whereas its fatiguability was less. Histochemical analysis showed atrophy of type I fibres in both diaphragm and gastrocnemius, associated with an increased proportion of type I fibres in the diaphragm only. Histological examination of both diaphragm and gastrocnemius showed

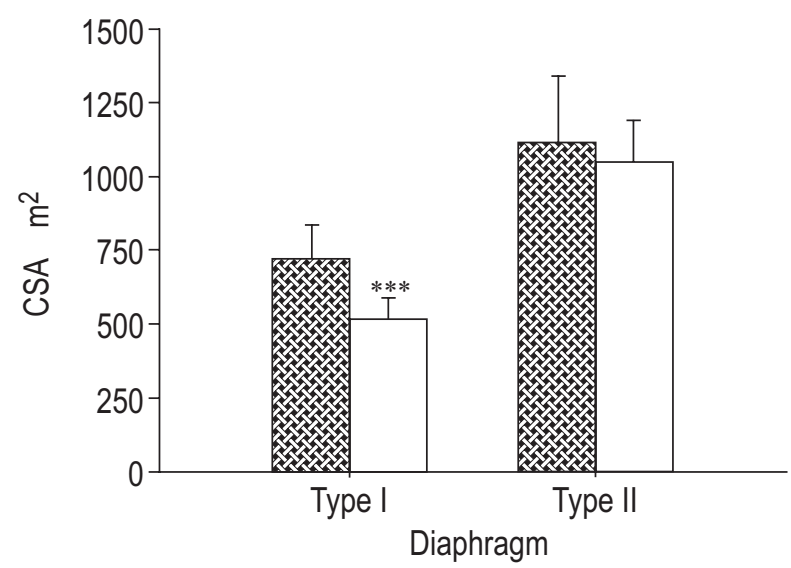

Fig. 5. - Diaphragm cross-sectional area (CSA) of type I and type II

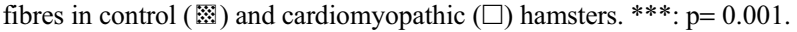

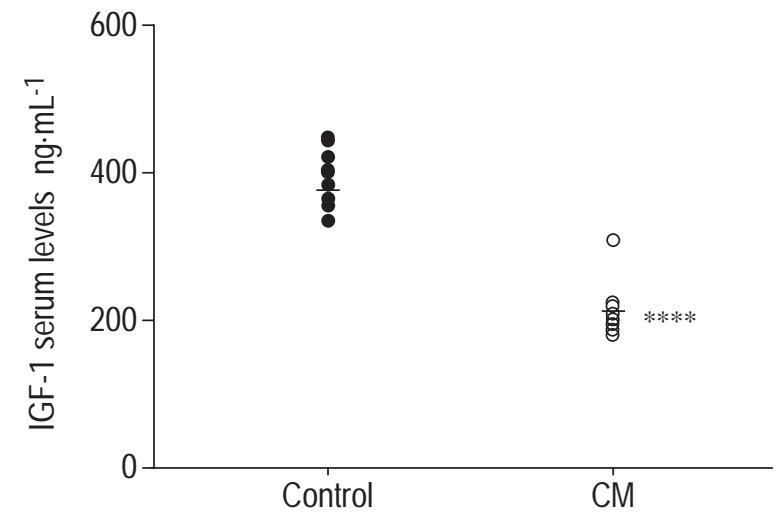

Fig. 6. - Serum levels of insulin-like growth factor (IGF)-1 in control (O) and cardiomyopathic $(\mathrm{CM} ; \mathrm{O})$ hamsters. The lines represent the mean values. $* * * *$ : $\mathrm{p}<0.0001$

a myopathic pattern in all fibre types. Finally, a significant decrease in IGF-1 serum levels, related to the diaphragm alterations, was observed in the CM group.

Cardiomyopathy in the Syrian hamster as a model of heart failure

A Syrian hamster model of dilated CM was chosen because pathophysiologically the dilated strain of $\mathrm{CM}$ appears to be more closely related to human CM than is the hypertrophic model [5]. Haemodynamic measurements in Syrian hamsters with dilated CM indicate cardiac failure at the age of 8-10 months [14], comparable to the age of the present hamsters. Clinical signs are clear only in the last stage of heart failure when congestion occurs [15].

Body, muscle and organ weight in dilated cardiomyopathy

The reductions found in body weight and heart and liver weight are comparable with the data on the Syrian hamster model in the literature [16, 17]. The unchanged absolute diaphragm weight and the increased diaphragm-to-body weight ratio are in contrast to the muscle wasting expected in dystrophic muscle. The finding is, however, in keeping with the data on diaphragm weight in the model of hypertrophic $\mathrm{CM}[6,18]$. In that model an increase in absolute diaphragm weight was even documented $[6,18]$. It presents, perhaps, a unique expression of the dystrophic process [6]. The finding of an unchanged absolute diaphragm weight is in contrast to the reduction in absolute gastrocnemius weight. This may be explained by the fact that the type I fibre atrophy in the diaphragm was associated with an increase in the number of type I fibres.

\section{Contractile properties of the diaphragm in dilated cardiomyopathy}

Several alterations in diaphragmatic contractile properties were noted in the present model of dilated CM. Firstly, at all frequencies a significantly lower force was observed in the diaphragm bundles of the CM group than in controls. Since force remained lower after correction for CSA, these 
findings are suggestive of myopathy. This was confirmed by histology (see below). Diaphragm weakness has also been reported with heart failure in humans $[19,20]$ as well as in different animal models of heart failure [3, 6, 21-23]. LECARPENTIER et al. [24] demonstrated a reduction in the number of cross-bridges in the diaphragm of hamsters with dilated $\mathrm{CM}$ as an explanation for its reduced force. The tension decline during the force-frequency curve might reflect muscle alterations rather than muscle fatigue. Alternatively, it is possible that the higher decline in tension observed during the force-frequency protocol in the CM group was dependent on the muscle fibre atrophy induced by $\mathrm{CM}$.

Secondly, significantly reduced diaphragm fatiguability was found in the CM group compared with controls, in contrast to the expected results. The present data do not offer a clear explanation for this apparent discrepancy. It is, however, possible that in CM animals the initial force reduction is so important that further decrease during the fatigue run is limited. To the authors' knowledge, diaphragm fatiguability has not been described in CM Syrian hamsters. Data in the literature concerning diaphragm fatiguability in CHF are controversial. On the one hand, in dogs with CEF induced by ventricular pacing [23], an increase in diaphragm fatiguability has been described. On the other hand, in Yucatan minipigs [3] with $\mathrm{CHF}$ after supraventricular pacing, it was shown to be unchanged.

Thirdly, the 1/2 RT appeared significantly reduced in $\mathrm{CM}$ animals. This is in contrast to the finding of a prolonged isotonic relaxation time in the diaphragm muscles of CM hamsters, demonstrated by CoIRAULT et al. [18]. Their model, however, concerns a hypertrophic model of CM. Moreover, in their study this impairment was clear only after preloading the muscle at $L$ o. The reduced $1 / 2 \mathrm{RT}$ may be explained by the type I fibre atrophy, knowing that these fibres are characterized by a reduced $\mathrm{Ca}^{2+}$ uptake and prolonged twitch relaxation time. An increased rate of sequestration of $\mathrm{Ca}^{2+}$ by the sarcoplasmic reticulum by means of a $\mathrm{Ca}^{2+}$ ATPase seems less likely. Indeed, ANGER et al. [17] found a decreased $\mathrm{Ca}^{2+}$ ATPase (SERCA 2) messenger ribonucleic acid (mRNA) level at 6 months of age in the CM Syrian hamster.

It is also possible that peripheral muscle dysfunction was present. No peripheral muscle contractile properties, however, were tested in this study.

\section{Skeletal muscle histology and histochemistry in dilated cardiomyopathy}

Histochemical evaluation revealed selective type I fibre atrophy in both gastrocnemius and diaphragm in combination with an increase in the number of type I cells in the diaphragm. Histochemistry has not previously been described in the model of dilated CM. In the model of hypertrophic $\mathrm{CM}$ [6] a reduction in fibre size in the diaphragm was clearly seen at 35 days and 180 days in type I and type II fibres, respectively. In contrast to the present findings, a fibre shift towards fast oxidative fibres was present. Differences in results may potentially be explained by differences in the progression of heart failure in the two models since in the hypertrophic model type I fibre atrophy is seen at an earlier stage. The described differences in histochemical findings between the two CM models confirm the suggestion of LiNDSAY et al. [4] that different models of heart failure affect the diaphragm in a different way.

The present histological data are in keeping with the myopathic pattern described in the diaphragm and limb muscles of patients with CM undergoing cardiac transplantation [4] and in the hypertrophic Syrian hamster strain [6]. They are also in line with the alterations in contractile properties found in the present study.

When the histological pattern of the diaphragm is compared with the gastrocnemius a similar atrophy pattern but with distinct fibre proportions are noted. This is in line with the findings of TIKUNOV et al. [25]. They described fast-to-slow transformations of both myosin and regulatory proteins, such as $\alpha$-tropomyosin and fast isoforms of troponin-T in the diaphragm of patients with CHF. This is in contrast to the increase in the number of fast fibres described in limb muscles [2]. It is possible that the different fibre proportion is due to the fact that in CHF the work of limb muscles tends to be decreased, whereas that of the diaphragm is increased [26].

Serum insulin-like growth factor-1 in dilated cardio-
myopathy

The finding of a reduction in serum IGF-1 is intriguing. IGF-1 is known to cause myofibre hypertrophy and plays an important role in skeletal muscle protein anabolism and growth [8]. The skeletal muscle changes seen in CM could be associated with the noted reduction in IGF-1 serum levels, which is likely to result from liver dysfunction associated with the CHF. Indeed, in the present study diaphragm alterations in contractile properties and histology were clearly related to the IGF-1 serum levels. This study suggests that reduced serum IGF-1 levels appear in heart failure independently of interfering factors such as medication. Whether the decrease in IGF-1 levels is associated with a downregulation of local muscle IGF-1 mRNA has to be examined. No hamster IGF-1 complementary deoxyribonucleic acid (cDNA) probe is, however, available and thus, the determination of IGF-1 expression in skeletal muscle remains problematic in these animals.

Are the myopathic changes documented in this model primary or secondary to heart failure?

A potential criticism of this model is that from the present study it cannot be determined whether the alterations found were primary, related to the congenital myopathy, or whether they were secondary to heart failure. The fact that heart failure could induce myopathic changes is, however, suggested by the study of SUPINSKI et al. [23]. They produced heart failure experimentally in dogs by ventricular pacing. In these dogs, the force of the diaphragm strips normalized to their surface area was decreased, suggesting a reduced quality of the diaphragm. Their study demonstrated that myopathic changes are conceptually possible in heart failure. It is, however, possible that some of the skeletal muscle changes found in the present animals are specific to the model of CM and cannot be generalized. Unfortunately, no data are available 
on the progression of histological changes in the model of dilated CM. In hypertrophic CM, JASMIN and PROSCHEK [27] described necrotic changes confined to the most active respiratory muscles in newborn animals. These changes extended progressively to the whole musculature with age, reaching their maximum severity in 100-day-old hamsters, and appeared in the heart after 40 days. This suggests that in hypertrophic $\mathrm{CM}$ animals at least some of the changes in skeletal muscles are due to the progressively developing myopathy rather than secondary to heart failure.

In conclusion, the present study confirms that heart failure in hamsters with dilated cardiomyopathy has an effect on the contractile and histological properties of the diaphragm. Type I fibre atrophy, in combination with an increase in the number of type I fibres, was associated with myopathic changes. This is in contrast to the model of hypertrophic cardiomyopathy, in which, at a similar age, type II fibre atrophy associated with a fibre shift towards fast oxidative fibres is seen. These differences in histochemical findings confirm the suggestion of LINDSAY et al. [4] that different models of heart failure affect the diaphragm in different ways. The reduced systemic production of insulin-like growth factor-1, related to the diaphragm changes, suggests a possible role for this growth factor in skeletal muscle abnormalities in cardiomyopathy.

\begin{abstract}
Acknowledgements. The authors thank N. Buts for expert technical help, E. Van Herck for IGF-1 measurement and A. Bisschop and R. Schepers for their help in statistical analysis.
\end{abstract}

\section{References}

1. Wilson JR, Mancini DM. Factors contributing to the exercise limitation of heart failure. $J$ Am Coll Cardiol 1993; 22: 93A-98A.

2. Drexler H, Riede U, Münzel T, König H, Funke E, Just H. Alterations of skeletal muscle in chronic heart failure. Circulation 1992; 85: 1751-1759.

3. Howell S, Maarek JI, Fournier M, Sullivan K, Zhan W, Sieck GC. Congestive heart failure: differential adaptation of the diaphragm and latissimus dorsi. J Appl Physiol 1995; 79: 389-397.

4. Lindsay DC, Lovegrove CA, Dunn MJ, et al. Histological abnormalities of muscle from limb, thorax and diaphragm in chronic heart failure. Eur Heart $J$ 1996; 17: 1239 1250 .

5. Whitmer JT, Kumar P, Solaro RJ. Calcium transport properties of cardiac sarcoplasmic reticulum from cardiomyopathic Syrian hamsters (Bio 53.58 and 14.6): evidence for a quantitative defect in dilated myopathic hearts not evident in hypertrophic hearts. Circ Res 1988; 62: 81-85.

6. Burbach JA, Schlenker E, Johnson JL. Morphometry, histochemistry, and contractility of dystrophic hamster diaphragm. Am J Physiol 1987; 253: R275-R284.

7. Järveläinen $H$, Rönnemaa T, Kallio V. Insulin-like growth factor-I in type 2 (non-insulin-dependent) diabetics with myocardial infarction and without macroangiopathy. Atherosclerosis 1986; 59: 335-340.

8. Froesh ER, Schmid C, Schwander J, Zapf J. Actions of insulin-like growth factors. Annu Rev Physiol 1985; 47: 443-467.

9. Dekhuijzen PNR, Gayan-Ramirez G, de Bock V, Dom R, Decramer M. Triamcinolone and prednisolone affect contractile properties and histopathology of rat diaphragm differently. J Clin Invest 1993; 92: 1534-1542.

10. Moore BJ, Miller MJ, Feldman HA. Diaphragm atrophy and weakness in cortisone-treated rats. $J$ Appl Physiol 1989; 67: 2420-2426.

11. Lewis MI, Monn SA, Zhan W, Sieck GC. Interactive effects of emphysema and malnutrition on diaphragm structure and function. J Appl Physiol 1994; 77: 947-955.

12. Close RI. Dynamic properties of mammalian skeletal muscles. Physiol Rev 1972; 52: 129-197.

13. Furlanetto RW, Marino JM. Radioimmunoassay of somatomedin C/insulin-like growth factor 1. Methods Enzymol 1987; 146: 216-226.

14. Panchal BC, Trippodo NC. Systemic and regional haemodynamics in conscious BIO TO-2 cardiomyopathic hamsters. Cardiovasc Res 1993; 27: 2264-2269.

15. Gertz EW. Cardiomyopathic Syrian hamster: a possible model of human disease. Prog Exp Tumor Res 1972; 16: 242-260.

16. Chemla D, Scalbert E, Desché P, Pourny J, Lambert F, Lecarpentier Y. Effects of perindopril on myocardial inotropy, lusitropy and economy, and on diaphragmatic contractility in the cardiomyopathic Syrian hamster. $J$ Pharmacol Exp Ther 1992; 262: 516-525.

17. Anger M, Lambert F, Chemla D, et al. Sarcoplasmic reticulum $\mathrm{Ca}^{2+}$ pumps in heart and diaphragm of cardiomyopathic hamster: effects of perindopril. Am J Physiol 1995; 268: H1947-H1953.

18. Coirault C, Chemla D, Pourny J, Lambert F, Riou B, Lecarpentier Y. Relaxation is impaired in the diaphragm muscle of the cardiomyopathic Syrian hamster. Am J Respir Crit Care Med 1997; 155: 1575-1582.

19. McParland C, Resch EF, Krishnan B, Wang Y, Cujec B, Gallagher CG. Inspiratory muscle weakness in chronic heart failure: role of nutrition and electrolyte status and systemic myopathy. Am J Respir Crit Care Med 1995; 151: 1101-1107.

20. Hammond MD, Bauer KA, Sharp JT, Rocha RD. Respiratory muscle strength in congestive heart failure. Chest 1990; 98: 1091-1094.

21. Lecarpentier $\mathrm{Y}$, Pery $\mathrm{N}$, Coirault $\mathrm{C}$, et al. Intrinsic alterations of diaphragm muscle in experimental cardiomyopathy. Am Heart J 1993; 126: 770-776.

22. Lecarpentier Y, Hervé P, Villeneuve A, Lacroix P, Duroux P. Alterations of in vitro diaphragm function during chronic heart failure in rabbit. Am Rev Respir Dis 1989; 139: A162.

23. Supinski G, Dimarco A, Dibner-Dunlap M. Alterations in diaphragm strength and fatiguability in congestive heart failure. J Appl Physiol 1994; 76: 2707-2713.

24. Lecarpentier Y, Coirault C, Lerebours G, et al. Effects of angiotensin converting enzyme inhibition on crossbridge properties of diaphragm in cardiomyopathic hamsters of the dilated Bio 53-58 strain. Am J Respir Crit Care Med 1997; 155: 630-636.

25. Tikunov B, Mancini D, Levine S. Changes in myofibrillar protein composition of human diaphragm elicited by congestive heart failure. J Mol Cell Cardiol 1996; 28: 2537-2541.

26. Mancini DM, Henson D, LaManca J, Levine S. Respiratory muscle function and dyspnea in patients with chronic congestive heart failure. Circulation 1992; 86: 909-918.

27. Jasmin G, Proschek L. Heriditary polymyopathy and cardiomyopathy in the Syrian hamster. I. Progression of heart and skeletal muscle lesions in the UM-X7.1 line. Muscle Nerve 1982; 5: 20-25. 